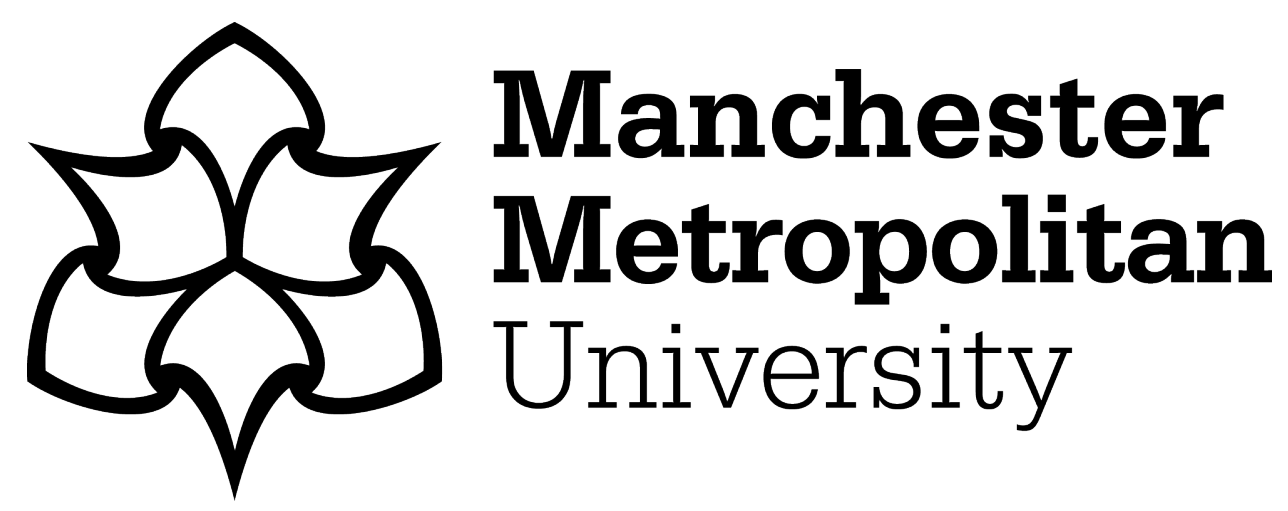

Hewitt, BM, Yap, Moi, Hodson-Tole, EF, Kennerley, AJ, Sharp, PS and Grant, Robyn (2017) A novel automated rodent tracker (ART), demonstrated in a mouse model of amyotrophic lateral sclerosis. Journal of Neuroscience Methods, 300. pp. 147-156. ISSN 0165-0270

Downloaded from: https://e-space.mmu.ac.uk/618345/

Version: Accepted Version

Publisher: Elsevier

DOI: https://doi.org/10.1016/j.jneumeth.2017.04.006

Usage rights: Creative Commons: Attribution-Noncommercial-No Derivative Works 4.0

Please cite the published version 


\title{
A novel automated rodent tracker (ART), demonstrated in a mouse model of amyotrophic lateral sclerosis
}

Brett M. Hewitt ${ }^{\mathrm{a}, \mathrm{b}}$, Moi Hoon Yap ${ }^{\mathrm{a}}$, Emma F. Hodson-Tole ${ }^{\mathrm{c}}$, Aneurin J Kennerley ${ }^{\mathrm{d}}$, Paul S. Sharp $^{\mathrm{d}}$, Robyn A. Grant ${ }^{\mathrm{b}^{*}}$

a. School of Computing, Mathematics and Digital Technology, Manchester Metropolitan University, Manchester, UK

b. School of Biology \& Conservation Ecology, Manchester Metropolitan University, Manchester, UK

c. School of Healthcare Science, Manchester Metropolitan University, Manchester, UK

d. Department of Psychology, University of Sheffield, Sheffield, UK

* Corresponding Author: Robyn.A.Grant: robyn.grant@mmu.ac.uk

\begin{abstract}
Background: Generating quantitative metrics of rodent locomotion and general behaviours from video footage is important in behavioural neuroscience studies. However, there is not yet a free software system that can process large amounts of video data with minimal user interventions.
\end{abstract}

New Method: Here we propose a new, automated rodent tracker (ART) that uses a simple rule-based system to quickly and robustly track rodent nose and body points, with minimal user input. Tracked points can then be used to identify behaviours, approximate body size and provide locomotion metrics, such as speed and distance.

Results: ART was demonstrated here on video recordings of a SOD1 mouse model, of amyotrophic lateral sclerosis, aged 30, 60, 90 and 120 days. Results showed a robust decline in locomotion speeds, as well as a reduction in object exploration and forward movement, with an increase in the time spent still. Body size approximations (centroid width), showed a significant decrease from P30.

Comparison with Existing Method(s): ART performed to a very similar accuracy as manual tracking and Ethovision (a commercially available alternative), with average differences in coordinate points of 0.6 and $0.8 \mathrm{~mm}$, respectively. However, it required much less user intervention than Ethovision ( 6 as opposed to 30 mouse clicks) and worked robustly over more videos.

Conclusions: ART provides an open-source option for behavioural analysis of rodents, performing to the same standards as commercially available software. It can be considered a validated, and accessible, alternative for researchers for whom non-invasive quantification of natural rodent behaviour is desirable.

Keywords: rodent behaviour, locomotion, image processing, software development, automated tracking 


\section{Introduction}

Generating quantifiable metrics for the assessment of animal health and welfare is often intrusive (such as taking physiological samples), and places the animal under a great deal of stress (Tieu 2011). Measurable behavioural models have been proposed as a way to mitigate a large part of the stress in rodents, and also allow for the animal to be studied while behaving freely (Tieu 2011). Several such behavioural models have been developed, including beam balancing (Stanley et al. 2005), rotorod (Hamm et al. 1994; Rozas et al. 1997), novel object investigation (Dudchenko et al. 2004; Antunes and Biala 2012), treadmill walking (Batka et al. 2014) and the morris water maze (Morris 1984, 2008). However, these tests require extensive animal training, and usually only measure the duration and frequency of certain behaviours, rather than true quantification of movements. Most laboratories will expose their animals to a host of these behavioural tests in order to capture a range of behaviours (Fraser et al. 2010). However, these approaches lack sufficient sensitivity to wholly characterise the progressions and recovery of animal health problems over different time frames (Casarrubea et al. 2015).

With improvements in video technology and associated software, it is now possible to collect footage from home-cage environments (Jhuang et al. 2010) with live streams and record large quantities of high-speed video footage (Nie et al. 2009). Video analyses of freely behaving rodents offer a robust way to non-invasively capture a range of quantifiable behavioural metrics, such as animal position and speed. They can also be more user-friendly than other methods, such as RFID tags, GPS or accelerometers (Koniar et al. 2016). Video analyses can be undertaken by the manual recording of behaviours and the manual tracking of certain points, such as the nose or tail point; however, this is a laborious process and is often subjective (Jhuang et al. 2010; Spink et al. 2001). Automation offers a robust alternative, as it does not suffer from fatigue, and can produce quantitative measurements of movements and behaviours (Spink et al. 2001).

To truly understand rodent locomotion and exploration, it is necessary to assess many aspects of behaviour by having a video analysis program that can both track animal movement and also categorise behaviours (Benjamini et al. 2010). There are a number of commercial rodent trackers, that are able to capture aspects of rodent movement in an open arena (i.e. Ethovision (Noldus) (Spink et al. 2001; Noldus et al. 2001), Any Maze (Ugo Basile), Videotrack (Viewpoint Behavior Technology) and Smart Video Tracking (Panlab, Harvard Apparatus)). These are usually able to measure distance travelled, speed of movement, position, and sometimes even identify the occurrence of certain behaviours, such as wall-following, or rearing (i.e. Ethovision (Noldus) (Spink et al. 2001; Noldus et al. 2001)). However, these programs tend to be expensive, require quite a lot of initial user input and are unable to be modified by users (i.e. are not open source) (Nema et al. 2016).

A variety of open source alternatives also exist; however, they tend to be quite specific and do not focus on measuring a range of locomotion and exploration behaviours. For example, they might only measure gait (Liang et al. 2012), track the rodent position (Zurn et al. 2005; Koniar et al. 2016), measure whiskers (Clack et al. 2012) or recognise specific postures (Farah et al. 2013; Ou-Yang et al. 2011). They also tend work with particular experimental laboratory arenas (OpenControl (Aguiar et al. 2007)) or home cage set-ups (Hong et al. 2015; Koniar et al. 2016). Many of these programs have also not been reliably validated against 
other methods or in other labs (Benjamini et al. 2010; Desland et al. 2014; Koniar et al. 2016), and require coding skills to adapt the code to other set-ups or extract alternative metrics (Nema et al. 2016). Ethowatcher (Junior et al. 2012) is an open source tracker, that can measure many aspects of animal movement and behaviour, however it still requires a lot of manual intervention during set-up. Therefore, despite advances in technology and tracking software, there does not yet exist a free, open source rodent tracker that requires little user interaction in order to process large quantities of behavioural data from video footage. Indeed, as technology continues to develop, current abilities to collect large video-based datasets are not matched by a concomitant ability to reliably and efficiently analyse such data volumes. Such mismatch in capabilities limits potential knowledge advances in associated fields and as such requires urgent attention to amend the current situation.

This paper, therefore, introduces a novel Automatic Rodent Tracker (ART), which can quickly and efficiently process large amounts of video data, with minimal user interactions. We validate the tracker's accuracy against manual tracking and Ethovision, and then demonstrate its capabilities on a dataset, comparing measures of movement and behaviour in SOD1-G93A mice to age-matched controls.

\section{Materials and Methods}

\subsection{Animals}

For this study, five female SOD $1^{\mathrm{G} 93 \mathrm{~A}}$ mice and five female control littermates were imaged in a 7 T magnet, at post-natal day (P) 30, P60, P90 and P120 ( \pm 3 days), to measure loss of pelvic muscle volume. They were also filmed in an open arena three days post-imaging. Mice were originally obtained from the Jackson Laboratory, B6SJL-Tg (SOD1-G93A)1Gur/J (stock number 002726), and subsequently backcrossed onto the C57B1/6 background (Harlan UK, C57Bl/6 J OlaHsd) for $>20$ generations (Mead et al. 2011). Our model, on a defined inbred genetic background, shows no effect of sex or litter of origin on survival (Mead et al. 2011). SOD $1^{\mathrm{G} 93 \mathrm{~A}}$ mice have an average survival of 140 days (Mead et al. 2011). None of the mice had undergone any scientific procedures prior to this study. All the animals were female kept on a 12:12 light schedule at $22{ }^{\circ} \mathrm{C}$, with water and food (standard laboratory rodent diet pellets) ad libitum. Mice were group-housed in standard rectangular cages $(48 \times 15 \times 13 \mathrm{~cm})$ containing a red Perspex shelter, with their littermates (2-6 animals per cage). SOD ${ }^{\text {G93A }}$ mice do suffer from weight loss, and the average body weights (mean \pm standard error) of the mice at each age can be compared in Figure 7a. All mice underwent welfare assessments on a bi-weekly basis, and daily from P100, to make sure they were behaving naturally (moving, grooming and socially interacting). All procedures were approved by the local Ethics Committee at the University of Sheffield and UK Home Office, under the terms of the UK Animals (Scientific Procedures) Act, 1986.

\subsection{Behavioural Data Collection}

High-speed digital video recordings were made using a Photron Fastcam PCI camera, recording at 500 frames per second, shutterspeed of $0.5 \mathrm{~ms}$, and resolution of $1024 \times 1024$. The camera was suspended from the ceiling, above a custom-built rectangular $(40 \mathrm{~cm} \times 40$ $\mathrm{cm})$ viewing arena with a glass floor, ceiling, and endwall. A Perspex block $(55 \mathrm{~cm} \mathrm{x} 55 \mathrm{~cm})$ was placed in the arena to encourage exploration of the space (Figure 1a). The mice were 
recorded at P30, P60, P90 and P120 on two consecutive days. Mice were tested throughout the day. They were individually removed from their home cage and introduced to the arena in a different order each day. The experimenter was blind to the condition of the animal at P30 and P60, but the symptoms were clear in the SOD1 ${ }^{\mathrm{G} 93 \mathrm{~A}}$ mice from P90. Video data was collected in near darkness and back-lit using a normal spectrum lightbox for illumination. Multiple 1.6 second video clips were collected opportunistically (by manual trigger) when the animal moved beneath the field of view of the camera. Approximately 12 videos were collected from each animal per filming day, which only took around 5-10 minutes.

\subsection{Magnetic resonance imaging}

$\mathrm{SOD} 1^{\mathrm{G} 93 \mathrm{~A}}$ mice are characterised by a large reduction in hind limb muscle volume from around P60 or P90, and many studies characterise this decrease by using MRI (Brooks et al. 2004; Grant et al. 2014; Marcuzzo et al. 2011; Mead et al. 2011). While ART is primarily designed for tracking rodent movement and behaviour, it does so by tracing the rodent body shape (see section 2.4.3), which means it has the capacity to approximate a body area or width measurement. This is especially useful to measure changes in body shape, which would be useful for a number of mouse models, including the SOD $1^{\mathrm{G} 93 \mathrm{~A}}$ mice. Therefore, all the mice were blind-imaged in a $7 \mathrm{~T}$ magnet (Bruker BioSpecAVANCE, $310 \mathrm{~mm}$ bore, MRI system B/C 70/30), with pre-installed 12 channel RT-shim system (B-S30) and fitted with an actively shielded, $116 \mathrm{~mm}$ inner diameter, water cooled, 3 coil gradient system (Bruker BioSpin MRI GmbH B-GA12. $400 \mathrm{mT} / \mathrm{m}$ maximum strength per axis with $80 \mu \mathrm{s}$ ramps) to assess pelvic muscle volumes at the 30,60,90 and 120 day time points. Animals were weighed and then placed in a custom built Perspex magnet capsule and imaged under gaseous anaesthesia ( $1-1.5 \%$, flow rate $0.8-1.0 \mathrm{~L} / \mathrm{min}$ continuous inhalation through a nose cone). Anaesthetic level was controlled on the basis of respiratory parameters; monitored using a pressure sensitive pad placed under the mouse's chest (SAII Model 1025 monitoring and gating system). Inside the capsule, a non-magnetic ceramic heated hot air system (SAII MR-compatible Heater System for Small Animals) and rectal probe, integrated into the physiological monitoring system maintained the temperature of the animal. All animals were euthanized at the 120 day time point. A $1 \mathrm{H}$ birdcage volume resonator (Bruker, $300 \mathrm{MHz}, 1$ $\mathrm{kW}$ max, outer diameter $114 \mathrm{~mm} /$ inner diameter $72 \mathrm{~mm}$ ), placed at the iso-centre of the magnet was used for both RF transmission and reception. A workstation configured for use with ParaVisionTM 4.0 software operated the spectrometer. Following field shimming, offresonance correction and RF gain setting a tri-plane FLASH sequence (TR $=100 \mathrm{~ms}, \mathrm{TE}=6$ $\mathrm{ms}$, Flip angle $=30^{\circ}, \mathrm{Av}=1, \mathrm{FOV}=40 \mathrm{~mm} \times 40 \mathrm{~mm}$, Slice thk $=2 \mathrm{~mm}$, Matrix $=128 \times$ 128 ) was used for subject localisation. From this a fast ( $\sim 5 \mathrm{~min}) 3 \mathrm{D}$ FISP sequence (TR $=$ $8 / 1200 \mathrm{~ms}, \mathrm{TE}=4 \mathrm{~ms}, \mathrm{FOV}=40 \mathrm{~mm} \times 40 \mathrm{~mm} \times 40 \mathrm{~mm}$, Matrix $=256 \times 256 \times 128)$ allowed low SNR visualisation of the pelvic area and thus planning of 21 axial high SNR single Spin Echo images $(\mathrm{TR}=3200 \mathrm{~ms}, \mathrm{TE}=7.5 \mathrm{~ms}, \mathrm{Av}=1, \mathrm{FOV}=40 \mathrm{~mm} \times 40 \mathrm{~mm}$, Slice thk $=1 \mathrm{~mm}$, Matrix $=256 \times 256$ ) covering the entire lower hind limb and pelvic region.

\subsection{Video Tracking using ART}

The Automatic Rodent Tracker (ART, available from:

http://tracker.bretthewitt.net/downloads.php) is a standalone windows executable, developed using C\# v4.0, Windows Presentation Foundation (WPF) and the .NET framework v4.5, which allows for easy deployment and setup on PC's running windows 7/8/10 without any 
other libraries/software packages being required. It was developed to batch process large numbers of high quality video files, and provide measurements of locomotion (such as speed and position) and identify behaviours (such as exploration, moving forward, turning), with minimal user interaction. Only one setting is required from the user prior to execution, and error feedback is provided post-analysis, with the option to re-analyse specific videos with a different setting. ART uses existing techniques, such as convex hull (Efron 1965; Ahmad et al. 2014), hull bending, Canny edge detection (Canny 1986) and skeletonisation (Manzanera et al. 1999), alongside a number of rule-based algorithms, which are detailed further below. The tracker itself is modular and open source, enabling other modules to be developed and added to its framework; for instance, future automatic tracking modules, such as for whisker tracking or gait analysis, providing a means for it to be developed by and for the wider research community.

\subsubsection{Detection of the mouse and other objects}

Firstly, videos were saved from the camera as Audio Video Interleaved (.avi) format and all videos were loaded to the Automatic Rodent Tracker (ART) for analysis via batch processing. The tracker firstly identified the presence of a mouse by using background subtraction to find moving parts within the image sequences (Figure 1b) in each video frame. A minimum convex hull (Efron 1965; Ahmad et al. 2014) was used to enclose the mouse and was adapted by using hull bending to better fit the shape of the mouse in each frame. The enclosed mouse was subtracted from the frame to predict the background. Canny edge detection (Canny 1986) was then used to detect the background contours, including the edges of the Perspex arena, the Perspex block and other contours, like sawdust or faecal material within the arena. A binary background image was then created (Figure 1c), which allowed all background objects to be subtracted from each frame, leaving only the mouse visible (yellow contour in Figure 1d). The proposed method enabled the detection of the mouse and is robust to occlusion or when the mouse is interacting with other objects (Figure 1d).

\subsubsection{Detection of the mouse head}

Once the mouse and edges of the arena were identified, the tracker locates the head and body of the mouse using a proposed rule-based method (termed here as Rule Based Sliding Keypoints, RBSK) applied to every frame of the video. This method generates a set of keypoints around the contours (in yellow in Figure 2c). The distances (d) of the keypoints were determined dynamically to cater for videos of different resolutions. These keypoints were then rotated, or slid, around the image and a set of rules used to determine the position of the mouse head in each frame. The number of slides was dependant on the gap distance $d$. To determine the location within 2 pixels, with a gap distance of 40 pixels, 20 slides were used $\left(\frac{40}{2}=20\right)$.

Five key points, separated by a distance, $\mathrm{d}$, were used to identify a mouse head ( $\left.\mathrm{Px}_{0-4}, \mathrm{Py}_{0-4}\right)$ (Figure 2a). The position of these points, and thus the position of the mouse head, was determined by the following rules:

1) When a line is drawn between points $\mathrm{P}\left(\mathrm{x}_{1}, \mathrm{y}_{1}\right)$, and $\mathrm{P}\left(\mathrm{x}_{3}, \mathrm{y}_{3}\right)$, points $\mathrm{P}\left(\mathrm{x}_{0}, \mathrm{y}_{0}\right)$, and $\mathrm{P}\left(\mathrm{x}_{4}, \mathrm{y}_{4}\right)$ must lie on one side of the line, and point $\mathrm{P}\left(\mathrm{x}_{2}, \mathrm{y}_{2}\right)$ on the other (Figure $2 \mathrm{~b}$ ). 
2) The area of the triangle enclosed by points $\mathrm{P}\left(\mathrm{x}_{1}, \mathrm{y}_{1}\right), \mathrm{P}\left(\mathrm{x}_{2}, \mathrm{y}_{2}\right)$ and $\mathrm{P}\left(\mathrm{x}_{3}, \mathrm{y}_{3}\right)$ (Figure 2a) has a threshold defined as half the maximum possible area of a triangle $\left(\frac{\sqrt{3}}{4} \times d^{2}\right)$. This excludes potential locations that have a thin base, such as a leg.

3) The line drawn between points $\mathrm{P}\left(\mathrm{x}_{1}, \mathrm{y}_{1}\right)$ and $\mathrm{P}\left(\mathrm{x}_{3}, \mathrm{y}_{3}\right)$ (bottom of the triangle in Figure 2a) must cover black pixels. As the algorithm is predominantly based on contour matching, this ensures the potential object is a mouse, and not a "mouse looking" shape that has been created from a stretched leg, or other item.

4) The distance between points $\mathrm{P}\left(\mathrm{x}_{0}, \mathrm{y}_{0}\right)$ and $\mathrm{P}\left(\mathrm{x}_{4}, \mathrm{y}_{4}\right)$ (Figure $\left.2 \mathrm{a}\right)$ must fall within a certain range, and is used to define the shape of the mouse. A lower bound of $1.2 \times \mathrm{d}$ and an upper bound of $2.5 \times \mathrm{d}$ is used to prevent the wide objects (such as the mouse rear) or narrow objects (such as a foot) being identified as a head.

5) The average intensity of the area defined by points $\mathrm{P}\left(\mathrm{x}_{1}, \mathrm{y}_{1}\right), \mathrm{P}\left(\mathrm{x}_{2}, \mathrm{y}_{2}\right)$ and $\mathrm{P}\left(\mathrm{x}_{3}, \mathrm{y}_{3}\right)$ must fall below a threshold intensity of 10 for a 256 bit image. This identifies the dark area of the mouse's head, rather than a similarly-shaped area (such as the * in Figure 2c).

6) The most accurate position of the head occurs when a keypoint coincides with the nose tip of the mouse, which is defined when the distance between the point $\mathrm{P}\left(\mathrm{x}_{2}, \mathrm{y}_{2}\right)$ and the line drawn between $\mathrm{P}\left(\mathrm{x}_{1}, \mathrm{y}_{1}\right)$ and $\mathrm{P}\left(\mathrm{x}_{3}, \mathrm{y}_{3}\right)$ (in green in Figure $2 \mathrm{c}$ ) is at a maximum.

\subsubsection{Defining the mouse body}

Once the head of the mouse had been found, it was combined with the earlier located mouse contour to define the whole shape of the mouse, along with the position and orientation of the head. When the whole body of the mouse is visible (Figure 3), a modified version of the RBSK was used to find both the head and tail tips (Figure 3), and skeletonisation (Manzanera et al. 1999) was performed to find the shortest route between the nose and tail tip, which generated a spine (Figure 3). The center of mass of the body segment was located, and a line drawn through this point, perpendicular to the spine and terminated at the outer contour. This line was termed the centroid width (Figure 3). This width measurement could be considered as a slice along the width of the animal, and was later correlated to aspects of body size, including body weight and pelvic muscle volume (Section 3.3.3).

\subsubsection{Extraction of behaviours and measures from the video}

All videos were uploaded to ART, and the program automatically selected trackable videos and frames. Trackable videos and frames were identified as when the mouse, and its head, was clearly in shot, and when the mouse was not rearing, which obscured the nose tip. This led to 726 videos being tracked during this experiment (10-17 videos per mouse, per age). ART can extract many variables from a video clip (Table 1). Firstly, the locomotion variables were extracted (Table 1), including average speed $(\mathrm{mm} / \mathrm{msec})$ and average angular speed (deg/ms). Maximum and minimum values of these variables could also be outputted by ART, per clip. Total distance travelled by the mouse, per clip, could also be produced, but was found to not be significantly affected by genotype in this study, so was excluded from further analysis.

General behaviour data could also be calculated from each clip, including the duration of time that the mouse moved forward, stayed still, turned and interacted with the Perspex block. The centroid width ( $\mathrm{mm}$ ) was also calculated (Table 1). The automatic calibration in ART was used to express distances, speeds and widths in mm. Video frame rate was 
extracted automatically from the video files; however, users could also change this manually. Due to ART being designed to be used on large datasets without user interaction, all metrics were outputted as per-clip averages (unless otherwise specified), with outliers (lowest and highest $10 \%$ ) removed. Metrics could also be outputted as per-mouse averages. All results were outputted from ART as .csv files, to allow easy integration with a number of analysis programs, including Matlab, SPSS and Excel, without the user having specialist programming knowledge. Raw tracked coordinate points could also be extracted as .csv files if the user did need to construct more complex analyses not yet incorporated in to the ART analysis suite.

Table 1: Summary of measures and behaviours extracted from the video by ART, including locomotion measures, general behaviours and size measures.

\begin{tabular}{|c|c|}
\hline Variable Name & Description \\
\hline \multicolumn{2}{|l|}{ Locomotion measures: } \\
\hline $\begin{array}{l}\text { Average Speed } \\
(\mathrm{mm} / \mathrm{msec})\end{array}$ & $\begin{array}{l}\text { Distance travelled by the centre of mass of the mouse (Fig. } 3 \text { ) per } \\
\text { frame, divided by time. Averaged for each clip, also outputs } \\
\text { maximum and minimum values. }\end{array}$ \\
\hline Average Angular & Tracked nose point and head key points used to calculate the \\
\hline Speed $(\mathrm{deg} / \mathrm{msec})$ & $\begin{array}{l}\text { angular distance the head moved per frame. Averaged for each } \\
\text { clip, also outputs maximum and minimum values. }\end{array}$ \\
\hline Total Distance $(\mathrm{mm})$ & $\begin{array}{l}\text { Total distance travelled by the centre of mass of the mouse, per } \\
\text { clip. }\end{array}$ \\
\hline General Behaviours: & Durations, allocated to frames when: \\
\hline Moving Forward & $\begin{array}{l}\text { Centre of mass of the mouse moved }>0.025 \mathrm{~mm} / \mathrm{msec} \text {, for at least } \\
80 \mathrm{~ms}\end{array}$ \\
\hline Staying Still & $\begin{array}{l}\text { Centre of mass of the mouse moved }<0.025 \mathrm{~mm} / \mathrm{msec} \text {, for at least } \\
80 \mathrm{~ms}\end{array}$ \\
\hline Turning & Nose point rotated $>0.2 \mathrm{deg} / \mathrm{ms}$ for at least $80 \mathrm{~ms}$ \\
\hline Interacting & Nose point came within $2-3 \mathrm{~mm}$ from the Perspex block. \\
\hline \multicolumn{2}{|l|}{ Size Measure: } \\
\hline Centroid width (mm) & $\begin{array}{l}\text { Tangent through the center of mass, normal to the spine (Fig. } 3 \text { ). } \\
\text { Only calculated when whole mouse body was visible and moving } \\
\text { forward to enable more reliable approximation. }\end{array}$ \\
\hline
\end{tabular}

\subsubsection{Validation of ART}

ART was validated against manual tracking and Ethovision XT 5.1.220 in a sub-set of 43 videos, containing clips of control and transgenic mice at each age (P30: $10 \mathrm{ntg}$, 8tg; P60: 5ntg, 5tg; P90: 3ntg, 5tg; P120: 6ntg, 1 tg). ART contains a validation option, so a user is able to validate the accuracy of ART for their set-up against a manual tracking example. In this instance, the accuracy of ART to find the nose point pixel coordinate (in red in Figure 3), was compared to manual tracking of the nose point in 43 videos, using the Manual Whisker Tracker (MWA) (Hewitt et al. 2016), by a trained and experienced experimenter. The error difference (in $\mathrm{mm}$ ) between the centre of the nose point in ART and MWA was calculated as an average in each of the 43 videos, over a period of 302-800 frames, and presented as a histogram. Manual tracking of the nose is considered the "ground truth" in this instance; however to investigate its reliability, five videos were tracked an additional time by the same experimenter to approximate manual error. In addition, a Bland-Altman plot (Bland \& 
Altman 1986) was constructed to compare manual tracking and ART in all the frames that contained nose tracking, in both the $\mathrm{x}$ and y planes.

ART was validated against Ethovision to observe how it compares generally to a commercial alternative. As Ethovision XT 5.1.220 did not find a nose point, but rather used the center of mass of the mouse body, this pixel coordinate was compared between ART and Ethovision XT 5, over the same 45 videos. The error difference (in $\mathrm{mm}$ ) between the centre of mass point in ART and Ethovision was calculated as an average in each of the 43 videos, over the same period of 302-800 frames. In addition, a Bland-Altman plot was constructed to compare Ethovision tracking and ART in all the frames that contained centre of mass tracking, in both the $\mathrm{x}$ and $\mathrm{y}$ planes. In both ART and Ethovision, speed, distance travelled and aspects of behaviour (resting/staying still and walking/moving forward) are all calculated from the centre of mass position; therefore a good agreement in this coordinate would also indicate a good agreement of all these parameters too. During the tracking set-up in ART and Ethovision, the minimum number of clicks out of the total number of 43 videos was also recorded, to estimate user intervention. Ethovision and ART have rather different tracking strategies, for example ART found both the nose point and the center of mass, while Ethovision only tracked the center of mass. Therefore, due to the additional processing steps in ART, running time was not compared between these programs.

\subsection{Statistical considerations}

All metrics were normally distributed, therefore parametric statistical tests were used throughout. A multivariate ANOVA was conducted on all the measures collated from the video footage. Average speed, average angular speed, centroid width and the percentages of time moving forward, turning, staying still and interacting were all inputted to the ANOVA as dependent variables. Genotype (SOD1 or control) and age (P30, P60, P90 and P120) were added as independent variables and Mouse ID as a covariate, to control for individual differences. Significant main and interaction effects were reported in the results to a significance level of $<0.05$, along with effect sizes (Partial Eta Squared: $\eta^{2} p$ ). Individual ANOVAs were then conducted as post hoc tests at each age, and indicated on the figures with an asterisk (*). Body weight and pelvic muscle volume were also examined using a multivariate ANOVA and were entered as dependent variables, with genotype and age as independent variables. As only one measure was collected per mouse for these variables, mouse ID did not need to be included in the analyses. Centroid width was also averaged, per mouse, and correlated to body weight and pelvic muscle volume using a Pearson's correlation.

\section{Results}

\subsection{Validation against manual tracking}

The average difference (error, mean $\pm \mathrm{sd}$ ) in the nose point coordinate between ART and a manual tracker was $0.60 \pm 0.24 \mathrm{~mm}$. This can be seen in Figure $4 \mathrm{e}$, which shows the average range of errors per clip in a histogram, with no error being larger than $1.4 \mathrm{~mm}$. The experimenter error in manually tracking the nose point coordinate was also calculated as $0.62 \pm 0.37 \mathrm{~mm}$. Figure $4 \mathrm{a}$ and $4 \mathrm{c}$ show a Bland-Altman plot of the agreement between manual tracking and ART, in the $\mathrm{x}$ and y plane, respectively. The vast majority of points fell around zero, and were well within 3 standard deviations of zero (dashed line in figures), 
especially in the x plane, which had a particularly low difference in tracking between the two methods (Figure 4a).

\subsection{Validation against Ethovision}

The average difference (error, mean $\pm \mathrm{sd}$ ) in the centre of mass coordinate between ART and Ethovision was $0.78 \pm 0.68 \mathrm{~mm}$ ( $\mathrm{n}=43$ videos). This can be seen in Figure $4 \mathrm{f}$, which shows the average range of errors, per clip, in a histogram, with no error being larger than $4.6 \mathrm{~mm}$. As well as the error, the minimum number of clicks it took to set up each tracking video was also recorded. Ethovision took a minimum of 30 clicks, whereas ART took a minimum of 6 clicks. Figure $4 \mathrm{~b}$ and $4 \mathrm{~d}$ show a Bland-Altman plot of the agreement between Ethovision and ART, in the $\mathrm{x}$ and y plane, respectively. The vast majority of points fell around zero, and were well within 3 standard deviations of zero (dashed line in figures). The difference in tracking between ART and Ethovision, was much lower than that of ART and Manual Tracking, for instance the 3 standard error lines (dashed lines) in Figure $4 \mathrm{~b}$ and $4 \mathrm{~d}$ were much lower than those in Figure 4a and 4c.

\subsection{Demonstration of ART on an experimental dataset of SOD1 and control mice}

\subsubsection{Locomotion was impacted in SOD1 mice.}

The average speed of locomotion was significantly reduced in SOD1 mice (Multivariate ANOVA Main Effect: $\left.F(1,327)=16.414, p<0.001, \eta^{2} p=0.049\right)$, at both P30 and P120 (Figure 5a). The SOD1 mice had a tendency to rotate more, indicated by elevated angular speeds at P60 and P90 (Figure 5b), however, this was not a significant finding (Multivariate ANOVA Interaction Effect: $\left.F(3,327)=2.4432, p=0.065, \eta^{2} p=0.022\right)$.

\subsubsection{General behaviours were altered in SOD1 mice}

Overall, SOD1 mice spent less time interacting with the Perspex block within the arena (Multivariate ANOVA Main Effect: $F(1,327)=4.762, p=0.030, \eta^{2} p=0.015$ ) and more time stood still (Multivariate ANOVA Main Effect: $F(1,327)=6.198, p=0.013, \eta^{2} p=0.019$ ) (Figure 6). In particular, at P120 SOD1 mice spent less time moving forwards (Multivariate ANOVA Interaction Effect: $\left.F(3,327)=6.508, p<0.001, \eta^{2} p=0.058\right)$ and more time stood still (Multivariate ANOVA Interaction Effect: $F(3,327)=7.209, p=<0.001, \eta^{2} p=0.058$ ) (Figure 6b). There were no significant effects of genotype or age on the percentage of time spent turning.

\subsubsection{Body size and weight were affected in SOD1 mice}

SOD1 mice weighed significantly less than control mice at P90 and P120 (Multivariate ANOVA Main Effect: $\left.F(1,327)=84.691, p<0.001, \eta^{2} p=0.738\right)$ (Figure 7a), and also had a reduced pelvic muscle volume at P60, P90 and P120 (Multivariate ANOVA Main Effect: $\left.\mathrm{F}(1,327)=251.874, \mathrm{p}<0.001, \mathrm{n}^{2} \mathrm{p}=0.894\right)$ (Figure $7 \mathrm{c}$ ). This can be seen clearly in the example MRI Pelvic Muscle images in Figure 7f. Centroid width was significantly reduced in SOD1 mice, in all age groups (Multivariate ANOVA Main Effect: $F(1,327)=201.733$, $\mathrm{p}<0.001, \eta^{2} \mathrm{p}=0.388$ ) (Figure 7e). Centroid width was also significantly correlated to both body weight (Pearson's Correlation: $r=0.766, p<0.001$ ) (Figure $7 b$ ) and pelvic muscle volume (Pearson's Correlation: $\mathrm{r}=0.420, \mathrm{p}=0.009$ ) (Figure 7d).

\section{Discussion}


We present here the Automatic Rodent Tracker (ART), and show that it i) performs similarly to manual tracking; ii) performs well compared to Ethovision; and iii) can extract important locomotion measurements, and behavioural classifications, from video data of freely behaving healthy and mutant mice.

\subsection{Validating and comparing ART}

ART performed well, in terms of accuracy, when it was compared to both manual tracking $(0.60 \pm 0.24 \mathrm{~mm})$ and Ethovision $(0.78 \pm 0.68 \mathrm{~mm})$ (Figure 4). These error values were particularly low, considering that the total viewing area was $40 \times 40 \mathrm{~cm}$. A critique of some past open-source trackers have been their lack of validation (Nema et al. 2016); ART overcomes this by containing a validation option that allows users to calculate error from manual tracking. In addition, in ART, the tracking was overlaid on to the video, and users could validate it frame-by-frame. In other trackers (such as Ethovision), the tracking is previewed as a line, and cannot be explored on a frame-by-frame basis in tandem with the position of the mouse. Manual tracking was used here as the "ground truth" of the nose point coordinate; however, when the experimenter manually tracked videos again, the nose point coordinate varied by around $0.62 \pm 0.37 \mathrm{~mm}$. This experimenter error was very low; however, automatic tracking further reduces errors of this type, and provides a more repeatable alternative (Spink et al. 2001).

As well as reducing manual error, automatic tracking should also reduce manual intervention. ART has much lower manual intervention scores per video analysed than Ethovision ( 6 clicks as opposed to 30), indicating an increased ease of setting up videos for analysis. In addition, ART can also batch-process videos, so large amounts of data can be processed at once further reducing demands on operator time. With the increases in the amount of video data collected, caused by recent technology developments (Jhuang et al. 2010; Nie et al. 2009) and a growing emphasis on behavioural data collection for animal welfare (Tieu 2011), it has become even more important for video analysis to be conducted quickly and in large batches with minimal user intervention. Furthermore, behavioural data is relatively variable, especially in SOD1 mice (Grant et al. 2014; Bucher et al. 2007), and it is recommended to have large animal numbers for all preclinical research, according to updated guidelines (Ludolph et al. 2010). This will continue to increase the amount of video data collected for behavioural studies, and the need for efficient analysis programs.

Automatic trackers vary in the way they process background and foreground images. ART uses a rule-based system that is quick to process. However, there are some instances where it does struggle to identify and track the mouse. One such instant is when the mouse rears, as the head changes shape significantly, and the nose point can become obscured (Figure 8a). Another example is if the mouse crosses a defined object (Figure 8b), for example if its head crosses over the Perspex block by climbing on top of it. ART primarily focusses on tracking the position and movement of the rodent head (such as its orientation and turning speed) and these measurements are not accurate if the mouse positions its head with severe pitch, roll and yaw; therefore, it is preferential for episodes of rearing and climbing to be omitted from any data processing of the head. If a user does need to make measurements when the rodent is rearing, an additional module could be added to future versions of ART to incorporate this. 
Ethovision also had some episodes where it did not track. Ethovision calculates the background image by processing one video frame that does not contain the mouse. This background has to be constant for all the subsequent frames, for instance, the camera view cannot move, the Perspex block cannot be moved, and the mouse cannot defecate nor brush sawdust in to the shot. If any of these things happen, then the mouse tracking becomes inaccurate (Figure 8c). ART overcomes this by processing the background in each individual frame, rather than just using one set frame. Another example where Ethovision had trouble tracking the footage was when the mouse got close enough to the Perspex arena wall that there was a reflection of the mouse appearing on the wall, and the refection was sometimes tracked instead of the actual mouse (Figure 8d). This was not a problem for ART, as a region of interest could be specified for processing within the arena walls, which omits areas of reflection. Indeed, we demonstrate here, that ART can robustly track a variety of videos with a very similar accuracy to costly proprietary software, such as Ethovision, with minimal user intervention.

It must be noted, however, that Ethovision is also constantly developing, and later versions (i.e. XT 12) now have the option to track multiple body points for rodents (including the nose and tail), as well as behavioural recognition options that can identify behaviours, such as walking, resting, rearing, sniffing and grooming (Desland et al. 2014). While the most recent version was not validated here, it does still require a number of manual inputs. We present ART here as a free, automated alternative, and hope that its modular and open nature will allow for further future developments to rival that of costly proprietary software. Indeed, open software has been shown to develop faster, with more functions added and defects corrected quicker, than closed-source software projects (Paulson et al. 2004).

\subsection{Demonstration of ART on experimental data}

We show here that locomotion speeds are significantly reduced in SOD1 mice at P30 and P120 (Figure 5). Other studies have also found that locomotion speeds are reduced in SOD1 mice. Grant et al. (2014) found that locomotion speeds were reduced in SOD1 mice from P30-P120, revealed by head-tracking of high-speed video footage, using an open source tracker, BWTT (Perkon et al. 2011). Only 4-8 videos were tracked per mouse in total in the previous study (compared to 42-69 here) as the BWTT required manual intervention to locate the nose and head points in one key frame (Perkon et al. 2011). Other behaviours, such as travelling and interacting could not be extracted from the BWTT tracker. Many studies have also examined locomotion from a gait analysis perspective by having mice walk on treadmills or catwalks (Wooley et al. 2005; Mancuso et al. 2011; Batka et al. 2014). However, data from these studies are highly dependent on the speed setting of the treadmill (Batka et al. 2014), and does not truly represent freely-moving rodent experiments. In addition, in order to measure other behaviours, such as rotating, interacting or staying still, requires an additional behavioural test, as these behaviours are unable to be carried out on a treadmill. There are not many studies that have looked at general behaviour changes in SOD1 mice (Batka et al. 2014), and the majority of studies have focussed on motor assessments, such as treadmill running, rotorod and grip strength (Batka et al. 2014). Therefore, the finding here, that SOD1 mice reduce their interaction (object exploration behaviours) overall, and especially at is a new result (figure 6). We also show that SOD1 mice are less active at P120, by staying still more and moving forward less. This agrees with other studies that have found activity levels 
and locomotion to be significantly impacted in these mice (Grant et al. 2014; Wooley et al. 2005; Mancuso et al. 201).

We also found a decrease in SOD1 body weight from P90 and a decrease in pelvic muscle volume from P60 (Figure 7a and c). While these are not new findings, and can also be found in other studies (Grant et al. 2014; Mead et al. 2011; Weydt et al. 2003), this is the first study to show that video analysis measurements of the centroid body width also correlated well with body weight (Figure $7 \mathrm{~b}$ ) and pelvic muscle volume, and was significantly reduced from P30 (Figure 7d). This approximation of size, using the centroid width, is a novel way to noninvasively monitor rodent health and, in the future, could replace having to directly handle the animal for weighing, or scanning the animal to extract muscle volumes.

Developing video analysis software, such as ART, allows many videos to be collected and processed from rodents behaving freely within a semi-natural environment. Not only this, but it characterises a number of quantifiable metrics of locomotion behaviour, general behaviours and rodent size measurements. This approach allows the collection of a variety of metrics, within the same behavioural set-up, thus decreasing the time of experimental data collection and the amount of animal handling.

\subsection{The ART Approach}

ART is a freely available, open source alternative to commercial rodent trackers, which performs at a very similar level of accuracy. It has been designed to be intuitive, easy to use, and require as little action as possible from the user. This is achieved by automating each step as much as possible. In addition, it uses the software design patterns and plug-in architecture (as described in Sherwood et. al. 2015), so that other behavioural trackers can be incorporated, such as gait analysis or whisker tracking. Future work will be to expand ART to include more behavioural metrics and to test it on a wide range of mouse models with motor deficits.

\section{Acknowledgements}

The authors would especially like to thank Prof Giuseppe Battaglia, Prof Tony Prescott and Dr Tennore Ramesh, all at the University of Sheffield 2009-2010, who helped to fund and plan the initial animal data collection (grants: BBSRC/EPSRC: EP/G062137/1, FET FP7 BIOTACT: ICT 215910). We are also extremely grateful to Emma Clear for all the manual tracking for the ART validation. Funding for this particular project was gained via an internally-funded PhD studentship, awarded to R.Grant from Manchester Metropolitan University.

\section{References}

Aguiar, P., Mendonça, L. and Galhardo, V., 2007. OpenControl: a free opensource software for video tracking and automated control of behavioral mazes. Journal of neuroscience methods, 166(1), pp.66-72. 
Ahmad, E., Yap, M.H., Degens, H. and McPhee, J.S., 2014, March. Atlas-registration based image segmentation of MRI human thigh muscles in 3D space. In SPIE Medical Imaging (pp. 90371L-90371L). International Society for Optics and Photonics.

Antunes, M. and Biala, G., 2012. The novel object recognition memory: neurobiology, test procedure, and its modifications. Cognitive processing, 13(2), pp.93-110.

Batka, R. J., Brown, T. J., Mcmillan, K. P., Meadows, R. M., Jones, K. J. and Haulcomb, M. M., 2014. The need for speed in rodent locomotion analyses. The Anatomical Record, 297(10), pp.1839-1864.

Benjamini, Y., Lipkind, D., Horev, G., Fonio, E., Kafkafi, N. and Golani, I., 2010. Ten ways to improve the quality of descriptions of whole-animal movement. Neuroscience \& Biobehavioral Reviews, 34(8), pp.1351-1365.

Bland, J. M., \& Altman, D. (1986). Statistical methods for assessing agreement between two methods of clinical measurement. The lancet, 327(8476), 307-310.

Brooks, K.J., Hill, M.D., Hockings, P.D. and Reid, D.G., 2004. MRI detects early hindlimb muscle atrophy in Gly93Ala superoxide dismutase-1 (G93A SOD1) transgenic mice, an animal model of familial amyotrophic lateral sclerosis. NMR in Biomedicine, 17(1), pp.28-32.

Bucher, S., Braunstein, K.E., Niessen, H.G., Kaulisch, T., Neurmaier, M., Boeckers, T.M., Stiller, D. and Ludolph, A.C., 2007. Vacuolization correlates with spin-spin relaxation time in motor brainstem nuclei and behavioural tests in the transgenic G93A-SOD1 mouse model of ALS. European Journal of Neuroscience, 26(7), pp.1895-1901.

Canny, J., 1986. A computational approach to edge detection. IEEE Transactions on pattern analysis and machine intelligence, (6), pp.679-698.

Casarrubea, M., Davies, C., Faulisi, F., Pierucci, M., Colangeli, R., Partridge, L., Chambers, S., Cassar, D., Valentino, M., Muscat, R. and Benigno, A., 2015. Acute nicotine induces anxiety and disrupts temporal pattern organization of rat exploratory behavior in hole-board: a potential role for the lateral habenula. Frontiers in cellular neuroscience, 9.

Clack, N.G., O'Connor, D.H., Huber, D., Petreanu, L., Hires, A., Peron, S., Svoboda, K. and Myers, E.W., 2012. Automated tracking of whiskers in videos of head fixed rodents. PLoS Comput Biol, 8(7), p.e1002591.

Desland, F.A., Afzal, A., Warraich, Z. and Mocco, J., 2014. Manual versus automated rodent behavioral assessment: Comparing efficacy and ease of bederson and garcia neurological deficit scores to an open field video-tracking system. Journal of central nervous system disease, 6, p.7.

Dudchenko, P.A., 2004. An overview of the tasks used to test working memory in rodents. Neuroscience \& Biobehavioral Reviews, 28(7), pp.699-709.

Efron, B., 1965. The convex hull of a random set of points. Biometrika, 52(3-4), pp.331-343.

Farah, R., Langlois, J.P. and Bilodeau, G., 2013. Catching a rat by its edglets. IEEE Transactions on Image Processing, 22(2), pp.668-678. 
Fraser, L.M., Brown, R.E., Hussin, A., Fontana, M., Whittaker, A., O’Leary, T.P., Lederle, L., Holmes, A. and Ramos, A., 2010. Measuring anxiety-and locomotion-related behaviours in mice: a new way of using old tests. Psychopharmacology, 211(1), pp.99-112.

Grant, R.A., Sharp, P.S., Kennerley, A.J., Berwick, J., Grierson, A., Ramesh, T. and Prescott, T.J., 2014. Abnormalities in whisking behaviour are associated with lesions in brain stem nuclei in a mouse model of amyotrophic lateral sclerosis. Behavioural brain research, 259, pp.274-283.

Hamm, R.J., PIKE, B.R., O'DELL, D.M., Lyeth, B.G. and JENKINS, L.W., 1994. The rotarod test: an evaluation of its effectiveness in assessing motor deficits following traumatic brain injury. Journal of neurotrauma, 11(2), pp.187-196.

Hewitt, B., Yap, M.H. and Grant, R., 2016. Manual Whisker Annotator (MWA): A Modular Open-Source Tool. Journal of Open Research Software, 4(1).

Hong, W., Kennedy, A., Burgos-Artizzu, X.P., Zelikowsky, M., Navonne, S.G., Perona, P. and Anderson, D.J., 2015. Automated measurement of mouse social behaviors using depth sensing, video tracking, and machine learning. Proceedings of the National Academy of Sciences, 112(38), pp.E5351-E5360.

Jhuang, H., Garrote, E., Yu, X., Khilnani, V., Poggio, T., Steele, A.D. and Serre, T., 2010. Automated home-cage behavioural phenotyping of mice.Nature communications, 1, p.68.

Junior, C.F.C., Pederiva, C.N., Bose, R.C., Garcia, V.A., Lino-de-Oliveira, C. and MarinoNeto, J., 2012. ETHOWATCHER: validation of a tool for behavioral and video-tracking analysis in laboratory animals. Computers in biology and medicine, 42(2), pp.257-264.

Koniar, D., Hargaš, L., Loncová, Z., Duchoň, F. and Beňo, P., 2016. Machine vision application in animal trajectory tracking. Computer methods and programs in biomedicine, 127, pp.258-272.

Liang, J.I., Chen, M.Y., Hsieh, T.H., Liu, C.Y., Lam, C.F., Chen, J.J.J. and Yeh, M.L., 2012. Video-based gait analysis for functional evaluation of healing achilles tendon in rats. Annals of biomedical engineering, 40(12), pp.2532-2540.

Ludolph, A.C., Bendotti, C., Blaugrund, E., Chio, A., Greensmith, L., Loeffler, J.P., Mead, R., Niessen, H.G., Petri, S., Pradat, P.F. and Robberecht, W., 2010. Guidelines for preclinical animal research in ALS/MND: A consensus meeting. Amyotrophic Lateral Sclerosis, 11(1-2), pp.38-45.

Mancuso, R., Oliván, S., Osta, R. and Navarro, X., 2011. Evolution of gait abnormalities in SOD1 G93A transgenic mice. Brain research, 1406, pp.65-73.

Manzanera, A., Bernard, T.M., Preteux, F.J. and Longuet, B., 1999, September. Unified mathematical framework for a compact and fully parallel $\mathrm{nD}$ skeletonization procedure. In SPIE's International Symposium on Optical Science, Engineering, and Instrumentation (pp. 57-68). International Society for Optics and Photonics.

Marcuzzo, S., Zucca, I., Mastropietro, A., de Rosbo, N.K., Cavalcante, P., Tartari, S., Bonanno, S., Preite, L., Mantegazza, R. and Bernasconi, P., 2011. Hind limb muscle atrophy 
precedes cerebral neuronal degeneration in G93A-SOD1 mouse model of amyotrophic lateral sclerosis: a longitudinal MRI study. Experimental neurology, 231(1), pp.30-37

Mead, R.J., Bennett, E.J., Kennerley, A.J., Sharp, P., Sunyach, C., Kasher, P., Berwick, J., Pettmann, B., Battaglia, G., Azzouz, M. and Grierson, A., 2011. Optimised and rapid preclinical screening in the SOD1 G93A transgenic mouse model of amyotrophic lateral sclerosis (ALS). PLoS One,6(8), p.e23244.

Morris, R., 1984. Developments of a water-maze procedure for studying spatial learning in the rat. Journal of neuroscience methods, 11(1), pp.47-60.

Morris, R.G., 2008. Morris water maze. Scholarpedia, 3(8), p.6315.

Nema, S., Hasan, W., Bhargava, A. and Bhargava, Y., 2016. A novel method for automated tracking and quantification of adult zebrafish behaviour during anxiety. Journal of Neuroscience Methods, 271, pp.65-75.

Nie, Y., Ishii, I., Yamamoto, K., Orito, K. and Matsuda, H., 2009. Real-time scratching behavior quantification system for laboratory mice using high-speed vision. Journal of realtime image processing, 4(2), pp.181-190.

Noldus, L.P., Spink, A.J. and Tegelenbosch, R.A., 2001. EthoVision: a versatile video tracking system for automation of behavioral experiments.Behavior Research Methods, Instruments, \& Computers, 33(3), pp.398-414.

Ou-Yang, T.H., Tsai, M.L., Yen, C.T. and Lin, T.T., 2011. An infrared range camera-based approach for three-dimensional locomotion tracking and pose reconstruction in a rodent. Journal of neuroscience methods, 201(1), pp.116-123.

Paulson, J.W., Succi, G. and Eberlein, A., 2004. An empirical study of open-source and closed-source software products. IEEE Transactions on Software Engineering, 30(4), pp.246256.

Perkon, I., Košir, A., Itskov, P.M., Tasič, J. and Diamond, M.E., 2011. Unsupervised quantification of whisking and head movement in freely moving rodents. Journal of Neurophysiology, 105(4), pp.1950-1962.

Rozas, G. and Garcia, J.L., 1997. Drug-free evaluation of rat models of parkinsonism and nigral grafts using a new automated rotarod test. Brain research, 749(2), pp.188-199.

Sherwood, T., Ahmad, E. and Yap, M.H., 2015. Formulating efficient software solution for digital image processing system. Software: Practice and Experience.

Spink, A.J., Tegelenbosch, R.A.J., Buma, M.O.S. and Noldus, L.P.J.J., 2001. The EthoVision video tracking system - a tool for behavioral phenotyping of transgenic mice. Physiology \& behavior, 73(5), pp.731-744.

Stanley, J.L., Lincoln, R.J., Brown, T.A., McDonald, L.M., Dawson, G.R. and Reynolds, D.S., 2005. The mouse beam walking assay offers improved sensitivity over the mouse rotarod in determining motor coordination deficits induced by benzodiazepines. Journal of Psychopharmacology, 19(3), pp.221-227. 
Tieu, K., 2011. A guide to neurotoxic animal models of Parkinson's disease.Cold Spring Harbor perspectives in medicine, 1(1), p.a009316.

Weydt, P., Hong, S.Y., Kliot, M. and Möller, T., 2003. Assessing disease onset and progression in the SOD1 mouse model of ALS. Neuroreport, 14(7), pp.1051-1054.

Wooley, C.M., Sher, R.B., Kale, A., Frankel, W.N., Cox, G.A. and Seburn, K.L., 2005. Gait analysis detects early changes in transgenic SOD1 (G93A) mice. Muscle \& nerve, 32(1), pp.43-50.

Zurn, J.B., Hohmann, D., Dworkin, S.I. and Motai, Y., 2005, January. A real-time rodent tracking system for both light and dark cycle behavior analysis. In Application of Computer Vision, 2005. WACV/MOTIONS'05 Volume 1. Seventh IEEE Workshops on (Vol. 1, pp. 8792). IEEE.

\section{FIGURES}

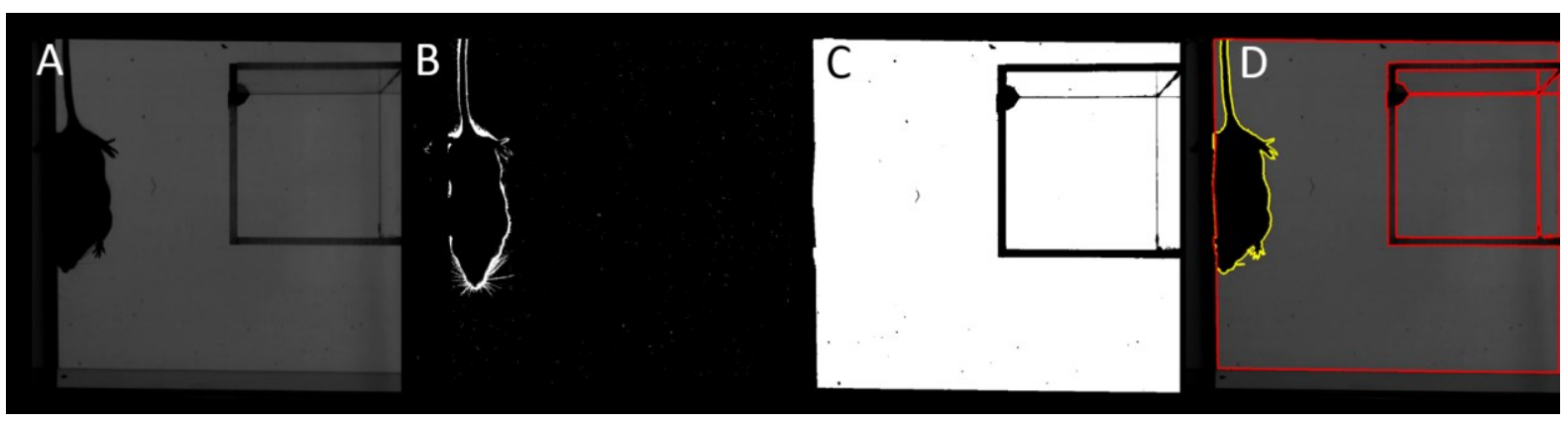

Figure 1: Processing of the videos on a per-frame basis. a) original image; b) background subtraction finds the moving mouse c) binary image of the predicted background, following preprocessing; d) contour image of the mouse in yellow, Perspex block and arena edges in red.

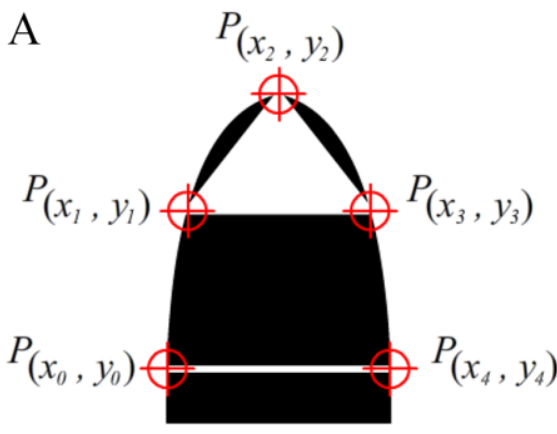

Figure 2: Five key points and rules defining the mouse head.

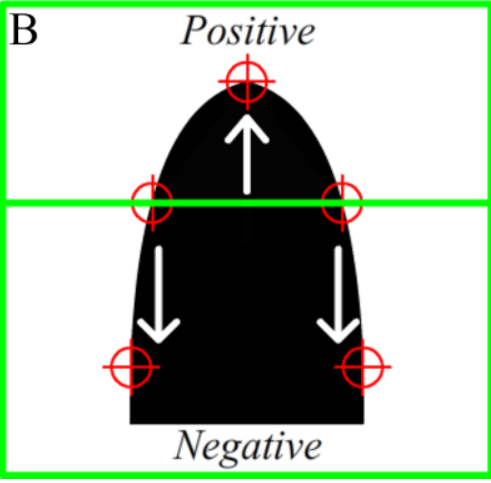

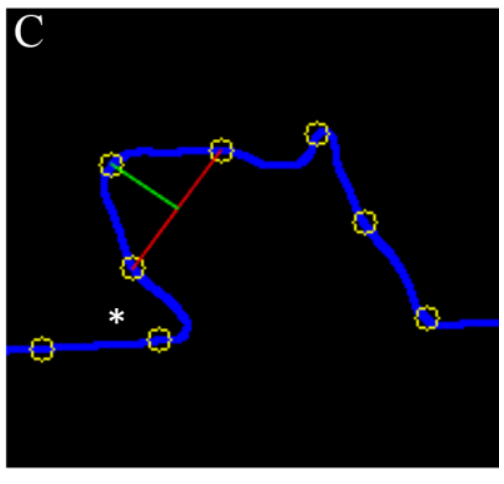




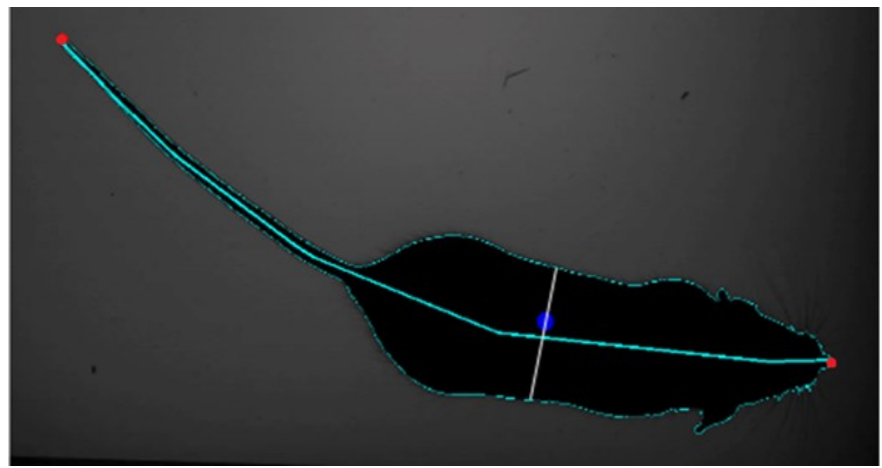

Figure 3: Whole mouse contour and spine. Spine is indicated by the light blue line, the white line indicated the centroid width. The nose and tail tips are shown by the red dots, and the center of mass of the body by the blue dot.

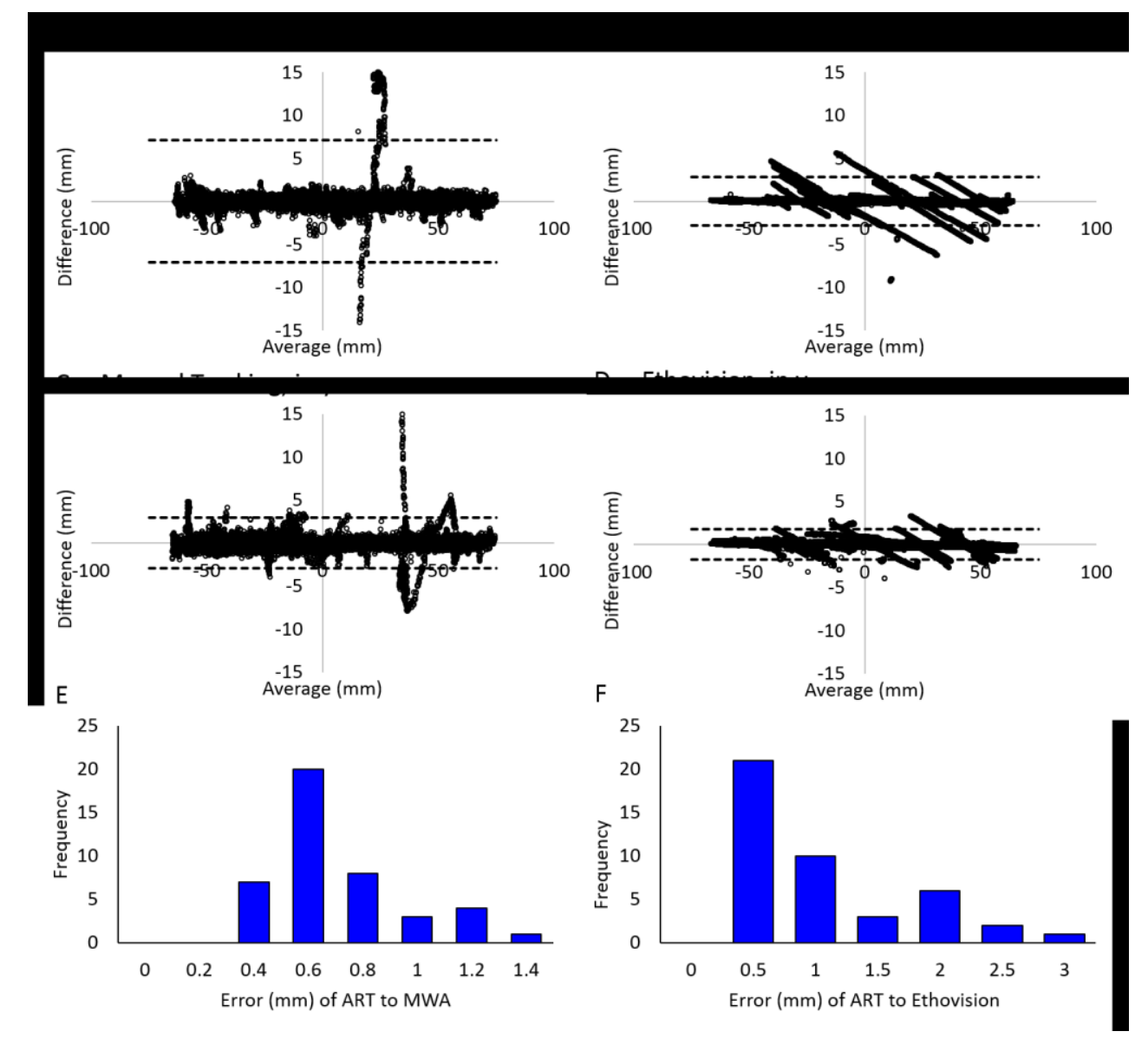

Figure 4: Validation of ART in terms of accuracy with manual tracking (MWA) and Ethovision. a) Bland-Altman plot comparing ART and manual tracking for the $\mathrm{x}$ coordinates of the nose point; $\mathrm{b}$ ) Bland-Altman plot comparing ART and Ethovision for the $\mathrm{x}$ coordinates of the centre of mass; $\mathrm{c}$ ) Bland-Altman plot comparing ART and manual tracking for the y coordinates of the nose point; $d$ ) Bland-Altman plot comparing ART and Ethovision for the y coordinates of the centre of mass. Dashed lines correspond to \pm 3 standard deviations. e) a histogram of error (in $\mathrm{mm}$ ) of the coordinate point of the nose, between ART and manual tracking; f) a histogram of error (in mm) of the coordinate point of the centre of mass, between ART and Ethovision. 


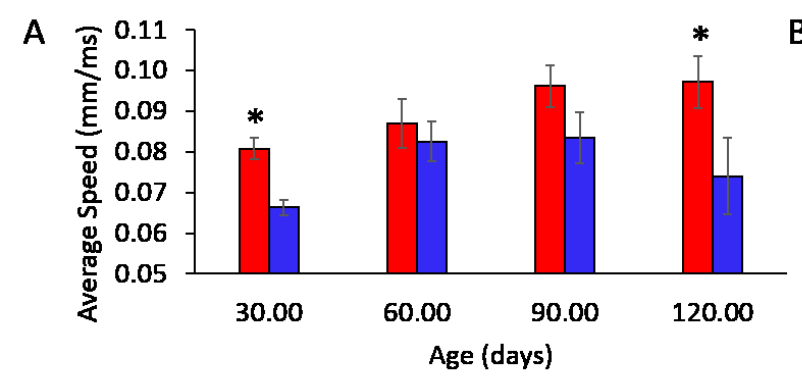

$\square$ Control $\square$ SOD1

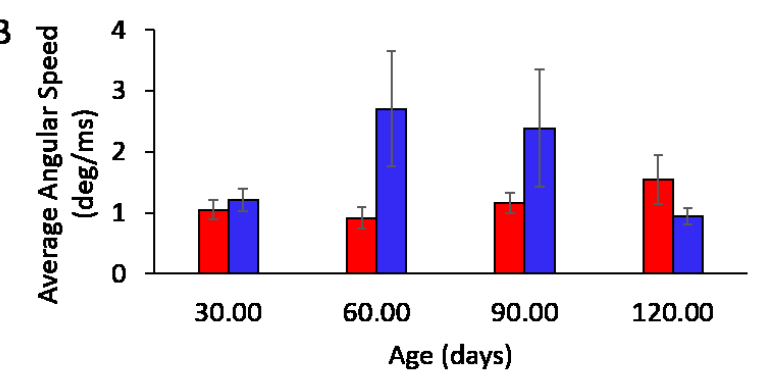

$\square$ Control $\square$ SOD1

Figure 5: Locomotion comparisons in SOD1 and control mice. a) Average locomotion speed was significantly slower in SOD1 mice at P30 and P120. b) Average angular (turning) speed was not significantly affected, despite some elevations at P60 and P90. Graphs show mean values, with standard error bars, significant interactions are indicated by an asterisk $(*)$.
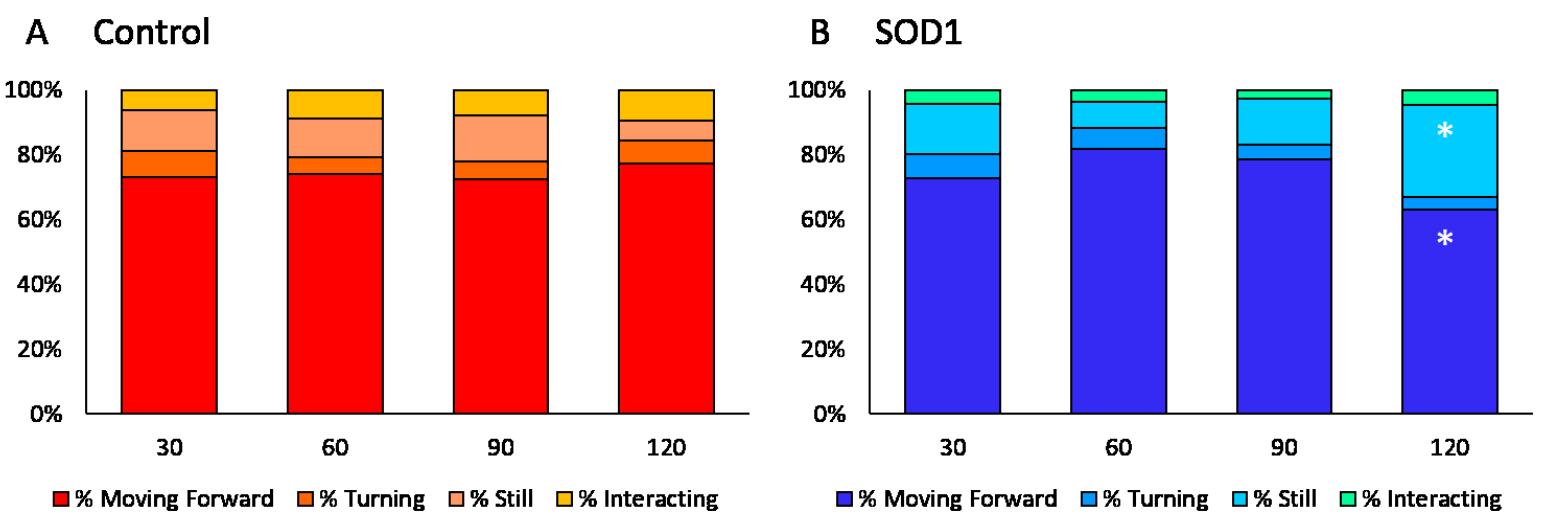

Figure 6: Percentage of time spent moving forward, turning, still and interacting in SOD1 (a) and control mice (b). Overall, SOD1 mice spent less time interacting with objects. At P120 SOD1 mice spent less time moving forwards and more time still. Graphs show the percentage time allocated to each of the defined behaviours, significant interactions are indicated by an asterisk $\left(^{*}\right)$. 


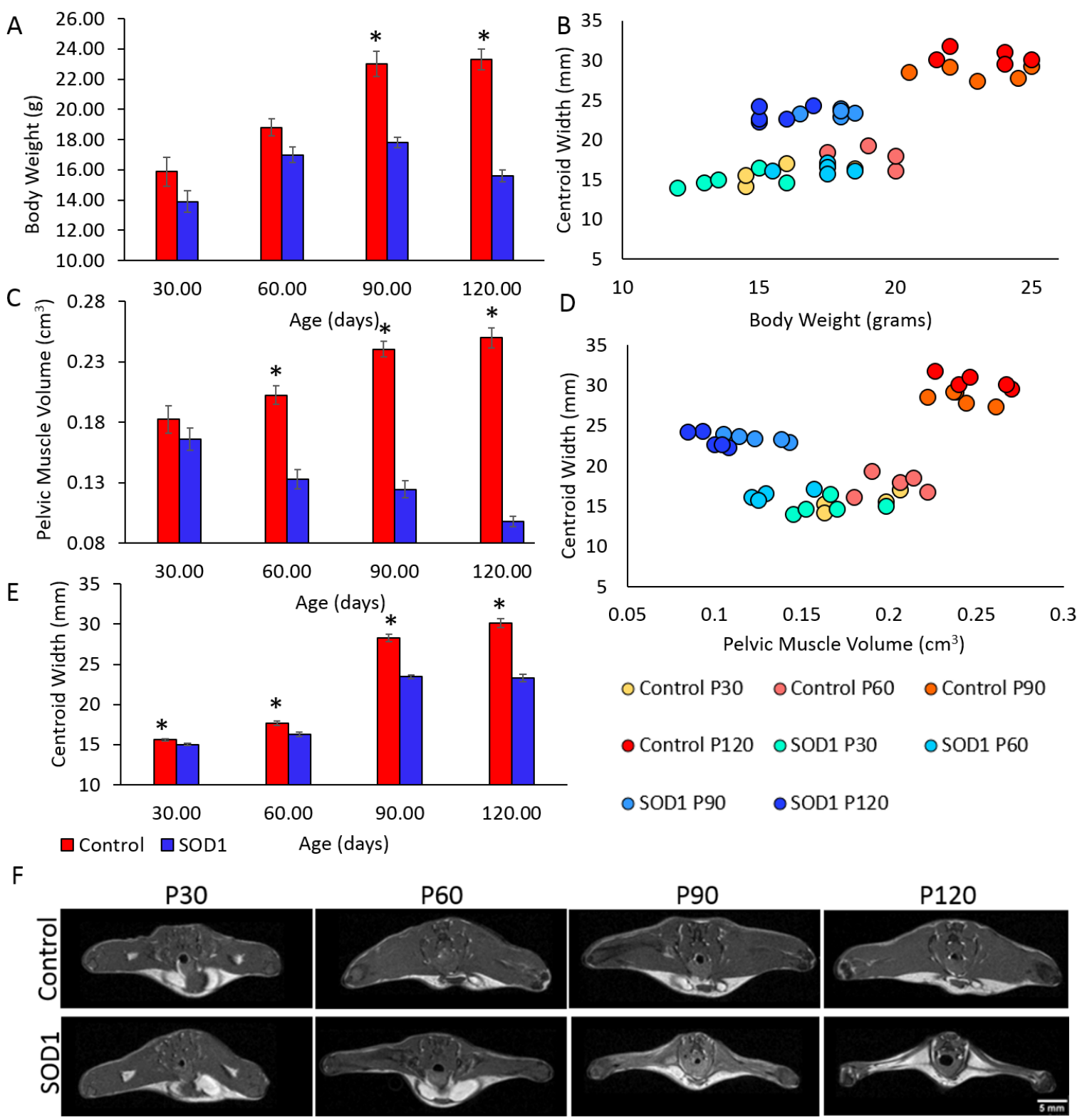

Figure 7: Body size and weight measurements in SOD1 and control mice. a) Body weight was significantly lower in SOD1 mice at P90 and P120. c) Pelvic muscle volume, taken from MRI measurements (an example shown in panel f) were reduced in SOD1 mice at P60, P90 and P120. e)

Centroid width, taken from the video, was lower in SOD1 mice throughout. Centroid width was significantly correlated to both body weight and pelvic muscle volume. a, $\mathrm{c}$ and e panels show mean values, with standard error bars, significant interactions are indicated by an asterisk $(*)$. b and d panels show a result per mouse, per age. 

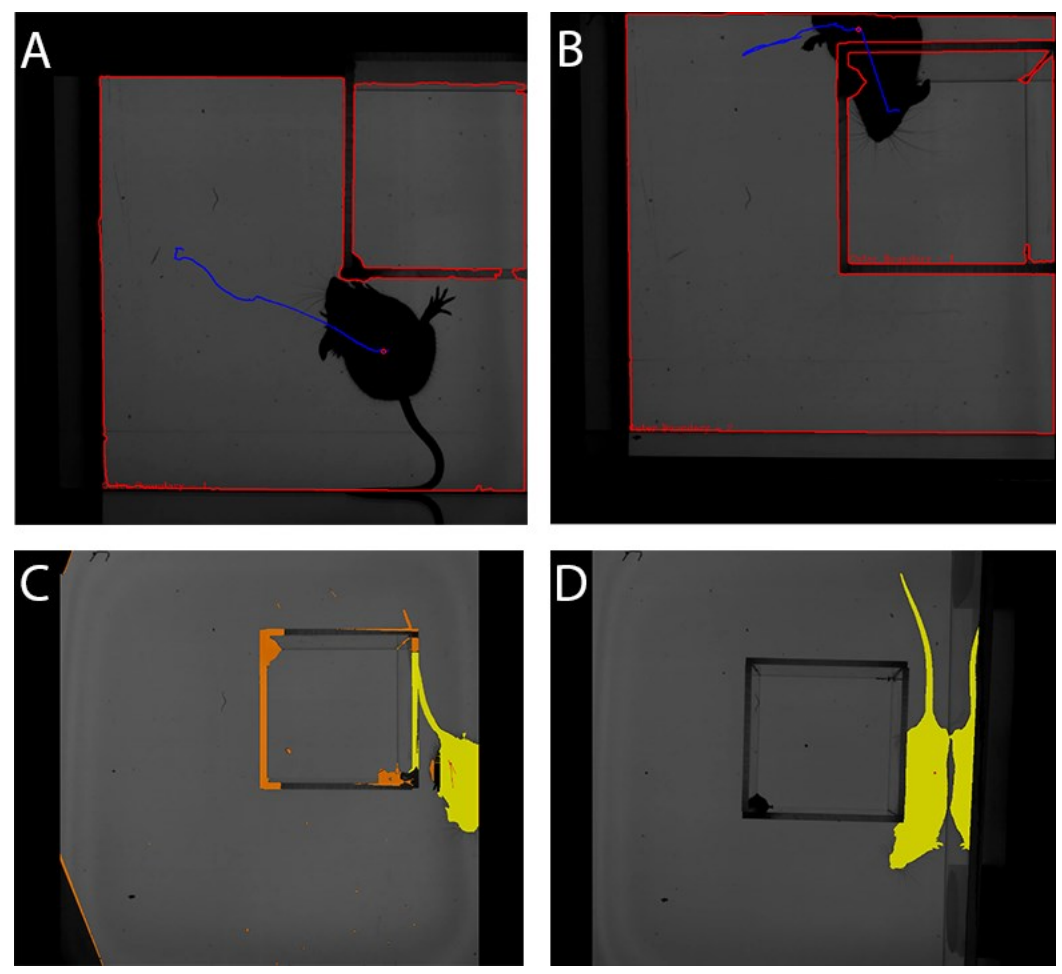

Figure 8: Failure modes for ART (a and b) and Ethovision (c and d). a) ART: Mouse rearing and deforming the head silhouette, preventing the nose tip from being detected. b) ART: Mouse climbing over a static object, background subtraction would then cause the mouse to appear as two separate entities. c) Ethovision: A video where the Perspex box was in a unique position and the program could not therefore find an accurate background image, therefore identified both mouse and object as mouse (in yellow). d) Ethovision: A mouse standing adjacent to its reflection, so both the mouse and its reflection was identified as the mouse (in yellow). 